\title{
Geldanlage: Immobilien als Alternative
}

\author{
P. Dahinden
}

Korrespondenz: Patrik Dahinden Dipl. Treuhandexperte FMH Services Treuhand, Geschäftsstelle Sursee Lizenznehmerin:

Partner Treuhand AG, Sursee Christoph-Schnyder-Strasse 46 CH-6210 Sursee Tel. 0419267045
Wie weiter an den Börsen? Diese Frage stellen sich nebst den Anlageberatern der Finanzinstitute, den Finanzanalysten sowie den institutionellen Anlegern auch zahlreiche private Anleger. Als Alternative ist eine Investition in Immobilien durchaus prüfenswert.

\section{Spare in der Zeit, ...}

Dass eine grosse Anzahl von Anlegern gegenwärtig in einer Warteposition verharrt, belegen die Meldungen von Banken über historische Rekordbestände auf Sparkonten. Laut der Statistik der schweizerischen Nationalbank erreichten diese Konten im vergangenen Januar mit 213 Milliarden Franken den höchsten Stand aller Zeiten. Umso erstaunlicher sind diese Zahlen unter dem Gesichtspunkt der Rendite, welche diese Konten momentan aufweisen. Der durchschnittliche Zinssatz bei den Kantonalbanken beträgt zurzeit gerade mal 0,8 Prozent. Rechnet man hier die Jahresteuerung und die Steuerbelastung auf diesen Anlagen ein, ist ein realer Wertverlust schon fast vorprogrammiert.

\section{Hypozins im Tief}

Statt sich mit diesen trüben Aussichten abzufinden, können Immobilien eine interessante Alternative für viele Anleger darstellen. Mit den momentanen historisch tiefen Hypothekarzinssätzen lässt sich bei Liegenschaften eine ansprechende Rendite erzielen, dies um so mehr, als auf dem Wohnungsmarkt die Leerstände bei Mietobjekten rückläufig sind. Der grosse Konkurrenzdruck im Baugewerbe macht es möglich, dass sich Neubauten nach wie vor günstig realisieren lassen.

\section{Investitionsentscheid}

Jede Investition muss unter den drei Gesichtspunkten Liquidität, Risiko und Rendite betrachtet werden. Eine Anlage in Immobilien sollte als langfristiger Entscheid betrachtet werden, da die investierten Mittel meist über längere Zeit gebunden sind. Das Risiko kann gegenüber Anlagen in Wertschriften eher besser abgeschätzt werden, zumal der reale Wert einer Immobilie auch geringeren Wertschwankungen unterliegt, als dies bei Wertschriften oft der Fall ist. Bei der Rendite kann die Berechnung der künftigen Nettorendite eher besser abgeschätzt werden.

\section{Berechnung der Eigenkapitalrendite}

Statt auf die oft erwähnte Bruttorendite abzustellen, empfiehlt es sich, der Eigenkapitalrendite (was bleibt unter dem Strich für den Investor) die nötige Aufmerksamkeit zu schenken. Das investierte Eigenkapital wird für diese Berechnung den Bruttomieterträgen abzüglich sämtlicher laufender Kosten, welche durch diese Investition anfallen (Heiz- und Nebenkosten, Unterhalt der Liegenschaft, Hypothekarkosten, Abschreibungen und Steuern), gegenübergestellt.

\section{Abschätzen der Risiken}

Als zu berücksichtigende Risiken kommen folgende Punkte in Betracht:

- mögliche Leerstände der Mieteinheiten;

- ausserordentliche Unterhaltskosten (in den laufenden Kosten nicht eingerechnet);

- Anstieg der Hypothekarzinsen (soweit nicht durch Festhypotheken über eine gewisse Zeit abgesichert);

- Preisrückgang am Immobilienmarkt (wobei aus heutiger Sicht eher mit einem Preisanstieg gerechnet wird).

Sofern die Gesamtheit dieser Risiken inklusive einer angemessenen Verzinsung mit der zu erwartenden Nettorendite aufgewogen wird, kann die Investition als empfehlenswert betrachtet werden.

\section{Wohneigentum versus Miete}

Nebst der Anlage in Mietobjekte kann auch bei kleineren Vermögen eine Investition in Immobilien Sinn machen. Der Entscheid für ein Eigenheim hängt neben diversen persönlichen Faktoren auch von der Gegenüberstellung der laufenden Kosten für das Wohneigentum im Vergleich zu der Höhe einer jährlichen Miete ab. Auch hier kann der momentan tiefe Hypothekarzinssatz den Entscheid zugunsten eines Eigenheims beeinflussen. 


\section{FMH SERVICES}

\section{Unsere Treuhandspezialisten}

Geschäftsstelle Muri b. Bern, Herr Peter Schneider, Thorackerstrasse 3, 3074 Muri bei Bern, Tel. 0319518840 . Fax 0319518839 - E-Mail: peter.schneider@fmhtreuhand.ch

Geschäftsstelle Ostermundigen, Herr Harry Huwiler, Bernstrasse 102, 3072 Ostermundigen 2, Tel. 0319390139 - Fax 0319390131 - E-Mail: harry.huwiler@fmhtreuhand.ch

Geschäftsstelle Olten, Herr Peter Senn, Baslerstrasse 32, 4603 Olten,

Tel. 0622059035 . Fax 0622059039 - E-Mail: peter.senn@fmhtreuhand.ch

Geschäftsstelle Basel, Herr Linus Cavegn, Hirzbodenweg 103, 4020 Basel,

Tel. 0613195121 . Fax 0613195252 . E-Mail: linus.cavegn@fmhtreuhand.ch

Geschäftsstelle Lohn-Ammannsegg SO, Herr Rolf Lehmann, Alte Bernstr. 53, 4573 Lohn-Ammannsegg, Tel. 0326775442 . Fax 0326775441 . E-Mail: rolf.lehmann@fmhtreuhand.ch

Geschäftsstelle Muri / AG, Herr Roland Bütler, Kirchenfeldstrasse 6, 5630 Muri, Tel. 0566640309 • Fax 0566645566 • E-Mail: roland.buetler@fmhtreuhand.ch

Geschäftsstelle Cham, Herr Guido Schmid, Alte Steinhauserstrasse 1, 6330 Cham, Tel. 0417486290 . Fax 0417486201 . E-Mail: guido.schmid@fmhtreuhand.ch

Geschäftsstelle Sursee, Herr Patrik Dahinden, Christoph-Schnyder-Strasse 46, 6210 Sursee, Tel. 0419267045 . Fax 0419267025 . E-Mail: patrik.dahinden@fmhtreuhand.ch

Geschäftsstelle Stans, Herr Marcel Helfenstein, Hansmatt 32, 6370 Stans,

Tel. 0416111821 • Fax 0416111820 . E-Mail: marcel.helfenstein@fmhtreuhand.ch

Geschäftsstelle Zürich-Wiedikon, Herr Christoph Lautenschlager, Steinstrasse 21, 8036 Zürich, Tel. 014571575 . Fax 014571516 . E-Mail: christoph.lautenschlager@fmhtreuhand.ch

Geschäftsstelle Winterthur, Herr Urs Gross, Ackeretstrasse 3, 8407 Winterthur, Tel. 0522240241 . Fax 0522223328 . E-Mail: urs.gross@fmhtreuhand.ch

Geschäftsstelle Weinfelden, Herr Adrian Hartmann, Marktplatz 6, 8570 Weinfelden, Tel. 0716228686 . Fax 0716228688 . E-Mail: adrian.hartmann@fmhtreuhand.ch

Geschäftsstelle Oberuzwil / SG, Herr Martin Brenner, Wiesentalstr. 22, 9242 Oberuzwil, Tel. 0719513066 . Fax 0719514771 . E-Mail: martin.brenner@fmhtreuhand.ch

Geschäftsstelle Speicher / St. Gallen, Herr Jürg Schmid, Zaun 55, 9042 Speicher, Tel. 0713442175 . Fax 0713443980 . E-Mail: juerg.schmid@fmhtreuhand.ch

Geschäftsstelle Au / SG, Herr Anibal Alghisi, Berneckerstrasse 9, 9434 Au, Tel. 0717401787 . Fax 0717401785 . E-Mail: anibal.alghisi@fmhtreuhand.ch

Geschäftsstelle Chur, p.Adr. Riedi Ruffner Theus AG, Poststrasse 22, 7002 Chur, Tel. 0812584646 - Fax 0812584647 . E-Mail: giorgio.cappellin@fmhtreuhand.ch

Geschäftsstelle Lausanne, Monsieur Michel Favre, Rue Pichard 20, 1003 Lausanne, Tel. 0213175454 . Fax 0213175453 . E-Mail: fiduciaire.favre@fmhfiduciaire.ch

Geschäftsstelle Genf, Monsieur Jean-Charles Terraz, rue Pedro-Meylan 4, 1208 Genf, Tel. 0227866870 • Fax 0227866659 . E-Mail: jeancharles.terraz@fmhfiduciaire.ch

Geschäftsstelle Sierre, Monsieur Jean-Pierre Chevalley, Avenue des Alpes 6, 3960 Sierre, Tel. 0274551675 . Fax 0274550644 . E-Mail: jeanpierre.chevalley@fmhfiduciaire.ch

Geschäftsstelle Martigny, Monsieur Gérald Claude, av. des Prés-Beudin 20, 1920 Martigny, Tel. 0277232009 . Fax 0277232001 . E-Mail: gerald.claude@fmhfiduciaire.ch

Geschäftsstelle Chiasso, Signor Franco Pozzi, Corso San Gottardo 89, 6830 Chiasso, Tel. 0916976080 . Fax 0916836826 . E-Mail: franco.pozzi@fmhfiduciaria.ch 